\title{
Reflexiones en Torno al Movimiento Corporal Humano desde una Perspectiva Multidimensional y Compleja
} Reflections on the Body Movement Human Complex and
Multidimensional Perspective

Floralinda García Puello ${ }^{1}$

Universidad Simón Bolívar

\section{RESUMEN}

Se necesita un nuevo paradigma, una nueva visión de la realidad que nos ayude a entender el movimiento corporal humano desde una perspectiva multidimensional y compleja. Se trata de pasar de una concepción mecanicista a una concepción holística de la realidad, lo cual requiere, a su vez, una transformación de nuestros pensamientos, percepciones y valores. El objetivo es reflexionar en torno a los conceptos de cuerpo y movimiento desde una perspectiva multidimensional y compleja, expresando la diferencia de esta perspectiva con la visión estrictamente etimológica ligada al modelo biomédico cartesiano. Se realizó una búsqueda en las bases de datos de documentos científicos, que permitieron una nueva mirada integradora hacia el estudio del movimiento corporal humano. Todo ello a partir de las referencias teóricas de diferentes investigadores y enfoques como el antropológico, el energético, el filosófico y ontológico. Teorías como la del movimiento continuo y el movimiento como sistema complejo, que están en consonancia con los aportes recientes de las diferentes ramas del saber, suministran una base para la reflexión sobre el estudio y el ejercicio de la Fisioterapia.

Palabras Clave: cuerpo, movimiento, multidimensional, complejo (Fuente: DeCS).

\begin{abstract}
We need a new paradigm, a new vision of reality that helps us to understand human body movement from a complex and multidimensional perspective. It involves moving from a mechanistic to a holistic conception of reality, which requires, in turn, a transformation of our thinking, perceptions, and values. The objective is to reflect on body and movement concepts from a complex and multidimensional perspective, showing the difference between this perspective and the strictly etymological vision linked to the Cartesian biomedical model. A search was carried out in the databases of scientific documents, which gave a new integrative look at the study of human body movement; all from the theoretical references of different researchers and approaches such as the anthropological, the energetic, the philosophical and the ontological. Theories, such as the one concerning continuous movement and movement as a complex system, which are consonant with the recent contributions from the different branches of knowledge, provide a base for reflection on the study and exercise of Physiotherapy.
\end{abstract}

Keywords: body, movement, multidimensional, complex (Source: MeSH, NLM).

Fisioterapeuta. Estudios en medicina tradicional china. Especialista en fisioterapia en ortopedia. Maestrante en Salud Pública. Correo electrónico: fgarcia@unisimonbolivar.edu.co 


\section{Introducción}

Todo se mueve y nada permanece y en el mismo río no nos bañamos dos veces.

\section{Heráclito (1)}

A mediados del siglo XIX y a partir de la revolución científica, la medicina sufre un gran cambio, pues deja de ser dominio de la filosofía. Se convierte así en Medicina Moderna y entra a formar parte de las Ciencias de la Naturaleza, con lo cual también el enfermo se convierte en un objeto medible. De este modo, el médico se interesa menos en su personalidad y ya no lo ve de una manera integral sino científica (2).

Es evidente que hoy en día los conceptos de ser humano, cuerpo y movimiento se centran en el dualismo gnoseológico y antropológico, que, según el campo biomédico del pensamiento cartesiano, separan el alma del cuerpo y el cuerpo del movimiento (3). Incluso, en este siglo, la visión mecanicista del cuerpo ha llegado a ser tan preponderante que la medicina misma se ha fragmentado en decenas de subespecializaciones, fraccionando de esta manera el cuerpo en partes cada vez más pequeñas y distanciadas. Es claro que la ciencia y la medicina moderna han mejorado la condición humana en un sinnúmero de frentes; sin embargo, la división entre la mente y el cuerpo está tan profundamente arraigada en el pensamiento científico moderno, que la mayoría de los médicos de Occidente saben mucho más sobre el comportamiento de las moléculas que del comportamiento de los seres humanos (4).

Las teorías esenciales sobre el mundo y el sistema de valores que están en la base de nuestra cultura actual se formularon en los siglos XVI y XVII. Éstas dieron a nuestra civilización occidental los rasgos que caracterizan la era moderna y se convirtieron en las bases del paradigma que la ha dominado durante los últimos trescientos años. No obstante, hoy existe en el mundo una crisis ideológica debido a que la mayor parte de la élite académica tiene una percepción limitada de la realidad, la cual resulta totalmente inadecuada para entender los principales problemas de nuestro tiempo. Pues, definitivamente, no es posible entender estos problemas con la metodología fragmentada que caracteriza a nuestras disciplinas académicas y a nuestras agencias gubernamentales.
El método de razonamiento analítico creado por Descartes quizá sea la principal contribución que se ha hecho a la ciencia moderna. Éste se ha convertido en una característica esencial del pensamiento científico moderno y ha demostrado su utilidad en el desarrollo de teorías científicas y en la realización de proyectos tecnológicos extremadamente complejos. Pero, justamente, este pensamiento ha propiciado una postura reduccionista de las disciplinas académicas, las cuales mantienen la convicción de que es necesario reducir los fenómenos complejos a sus partes constitutivas para lograr entenderlos. Por otro lado, la afirmación de Descartes respecto a que el concepto de cuerpo no incluye nada que pertenezca a la mente, y el de mente, nada que pertenezca al cuerpo, ha calado hondo en la civilización occidental y ha provocado grandes consecuencias en su comportamiento, como el actual culto al cuerpo "ideal". Esta misma posición ha impedido a los médicos considerar las dimensiones psicológicas de las enfermedades, y a los psicoanalistas ocuparse del cuerpo de sus pacientes. En definitiva, el paradigma racional ha provocado una infinita confusión sobre la relación entre la mente y el cuerpo (5).

Mirando otro aspecto de nuestro problema, el estudio del movimiento corporal humano no puede homologarse con el estudio del "hombre máquina", conformado por palancas, huesos y músculos. Pues, esta forma de alineación produce un hombre extraño ante su propio cuerpo y su capacidad fisiocinética, además de justificar la postura práctica del dualismo cartesiano (6). Desde ésta perspectiva, el movimiento se entiende en específico como el conjunto de contracciones musculares producidas, a su vez, por un conjunto de sistemas corporales que evidencian el desplazamiento de segmentos a nivel espacial. Así, el cuerpo y el movimiento se conciben como máquinas $\mathrm{y}$, a su vez, como el producto de éstas (3).

Se necesita, entonces, un nuevo paradigma, una nueva visión de la realidad que nos ayude a entender el movimiento corporal humano desde una perspectiva multidimensional y compleja. Esto requiere una transformación de nuestros pensamientos, de nuestras percepciones y de nuestros valores. Se trata de pasar de una concepción mecanicista a una concepción holística de la realidad. Estamos ante una crisis compleja y multidimensional en el orden mundial, que afecta todos los aspectos de nuestras vidas $\mathrm{y}$, en consecuencia, se requiere un cambio de pensamiento 
que nos permita encontrar soluciones verdaderamente trascendentales para los problemas de salud de la humanidad.

La perspectiva de la complejidad plantea que el conocimiento de cualquier fenómeno de la existencia humana no puede ser lineal, y está lejos de la relación causa-efecto. En palabras de Morin, el conocimiento es una aventura en espiral que tiene un punto de partida histórico pero que no tiene término, sino que por el contrario da círculos concéntricos permanentes (7). Estudiar el ser humano en movimiento requiere, así, de una visión holística que lo entienda como un ser bio-psico-antropo-socio-cultural. Esta visión holística pone fin a la mirada mecanicista, fragmentaria, separatista y especialista newtoniana-cartesiana del modelo biomédico. Es una perspectiva de unión entre diversas disciplinas, que busca la transcendencia del conocimiento y lograr de esta manera una mejor asistencia al individuo. Desde ella, el movimiento corporal humano además de movimiento es intención, instrumento de crecimiento, de maduración y de aprendizaje y por esto resulta tan importante para el desarrollo y la evolución humana. El cuerpo se asume como un referente social, un centro de atracción por la diversidad de las manifestaciones y la amplitud de los contextos en los cuales se desenvuelve (8).

En definitiva, las ciencias que se proponen estudiar el movimiento corporal humano deben considerar la complejidad de su objeto de estudio. En este marco, a través de este artículo de revisión, se pretende ofrecer una idea clara de las teorías que abordan el estudio del movimiento corporal humano desde una perspectiva multidimensional y compleja, teniendo claro que el cuerpo, como objeto de conocimiento, es inasible y que éste es semiótico. Es decir, que no es sólo un ente biológico sino un sistema de signos, un lenguaje en sí mismo, conformado tanto por las apariencias corporales como por sus movimientos, sus gestos y sus comportamientos aprendidos (9).

Con base en estos últimos planteamientos, el presente artículo también realiza una aproximación al significado del movimiento corporal humano, dando a conocer diversas conceptualizaciones sobre el cuerpomovimiento y sobre la importancia de reconocerlos como parte del contexto conceptual de nuestro saber disciplinar, a través del cual el humano se reconoce así mismo y establece relaciones con el otro y su entorno. De igual forma, se pretende argumentar sobre la necesidad de reconocer al ser humano en una perspectiva holística, como una totalidad en la cual cuerpo y mente conforman una unidad inseparable, que funciona como un sistema abierto en la interrelación consigo mismo y con su entorno (6). Así se comprenderá el movimiento corporal humano como el resultado de la interacción de múltiples e infinidad de factores.

\section{Desarrollo}

En concreto, para comprender el movimiento corporal humano, es necesario realizar un análisis histórico desde algunas concepciones filosóficas, físicas y anatómicas. Presentamos aquí, una aproximación teórica para el tema propuesto sin pretender, claro está, un análisis exhaustivo de las mismas, puesto que ello escapa a nuestro objetivo central.

\section{Paradigmas en torno al concepto de cuerpo y movimiento}

En la mayoría de investigaciones e intervenciones que se realizan en el área de la salud, se evidencia una concepción separatista respecto a cuerpo y mente. Por un lado, se construyen modelos que trasladan a la materialidad lo que se conoce por mente; por otro lado, se profundiza en explicaciones de corte estructural y funcional sobre las diferentes partes del cuerpo. Como se ha dicho antes, esta postura dualista puede entenderse como la consecuencia lógica del modelo biomédico cartesiano, el cual se ha convertido en el hito de la evolución occidental de las ciencias (3). Esta visión ha generado una crisis en el ejercicio médico actual, debido principalmente a la negación de la realidad corpórea del ser humano $\square$ paciente $\square$ en el sentido de la afectividad y humanidad, cuestión que a su vez está relacionada con la cultura y el ámbito social (2). Partiendo de esta premisa, resulta propicio aludir a diversas posturas paradigmáticas sobre la comprensión del cuerpo y el movimiento, con el ánimo de orientar al lector en relación a la evolución histórica del problema. 


\section{El hombre máquina. Dualismo cartesiano y movimiento corporal humano}

La famosa expresión de Descartes, "Pienso, luego existo", sugiere el lugar privilegiado que la razón ocupa en lo humano (10). Este extremismo de Descartes implica un menosprecio por la corporeidad y la sensibilidad, pues en esa frase la razón resulta imperante y el cuerpo queda relegado a ser el vehículo del alma. De hecho, en el método cartesiano, la primera certeza es la duda y con ello se niega la existencia del cuerpo, pues, a partir de allí, las sensaciones carecen de veracidad y el pensar es lo único que no puede separarse del ser. Para Descartes, sentir es una forma de pensar, pero su postura sugiere una división entre cuerpo y alma en la que resulta imposible un punto intermedio entre los elementos en cuestión (11).

Descartes deduce que la esencia de la naturaleza humana se halla en el pensamiento y que todo aquello que sea percibido con gran claridad y distinción es absolutamente cierto. Este filósofo basaba toda su visión de la naturaleza en la división fundamental entre pensamiento-mente y materia-cuerpo. Según él, el universo material es una máquina y sólo una máquina. En la materia no hay vida ni metas ni espiritualidad. Y esta imagen mecanicista de la naturaleza dominó la ciencia después de Descartes, marcando la pauta de las investigaciones científicas y sugiriendo la formulación de todas las teorías sobre los fenómenos naturales.

La visión cartesiana de los organismos vivos influenció decisivamente en la evolución de las ciencias humanas, pero fue Isaac Newton quien realizó el sueño cartesiano y completó la revolución científica. La física newtoniana, logro supremo de la ciencia del siglo XVII, estableció una teoría matemática del mundo que se convirtió en la base del pensamiento científico hasta mediados del siglo XX. Newton aunó los descubrimientos de sus predecesores y formuló las leyes generales del movimiento que rigen todos los objetos del sistema solar, desde las piedras hasta los planetas. Utilizó su nuevo método matemático para formular las leyes exactas del movimiento para todos los cuerpos en los que influye la fuerza de gravedad. Según su teoría, todos los fenómenos tenían una causa $\mathrm{y}$ un efecto determinado, $\mathrm{y}$, en principio,se podía predecir con absoluta certeza el futuro de cualquier parte del sistema si se sabía con todo detalle el estado en el que se hallaba en un momento determinado. A consecuencia de ésta idea, el mundo comenzó a ser considerado un sistema mecánico que podía describirse objetivamente, sin tomar en cuenta al observador humano, y esta descripción objetiva de la naturaleza se convirtió en el ideal de todas las ciencias (5).

Como hemos dicho, los organismos vivos entraban también en la visión mecanicista de Descartes. Según ello, los seres humanos están habitados por un alma racional que se conecta con el cuerpo mediante la glándula pineal, situada en el centro del cerebro. En cuanto al cuerpo humano, era imposible diferenciarlo de un animal-máquina. El filósofo francés explicó detalladamente la manera de reducir los movimientos y las funciones biológicas del cuerpo a simples operaciones mecánicas, a fin de demostrar que los organismos vivos eran meros autómatas (5).

La influencia del paradigma cartesiano en el pensamiento médico inspiró el llamado modelo biomédico. En éste, la medicina como ciencia natural se despojó de la filosofía y de la metafísica y se tornó científica. Así apareció la teoría biológica de la enfermedad y esta fue vista como un defecto de la máquina biológica. Por medio del reduccionismo físico-químico, experimental, los biólogos constituyeron la biología como una disciplina tan científica como la física y la química, considerando al organismo humano como una máquina compleja cuyo estudio se realiza mediante el aislamiento de sus partes (12).

Desde esta perspectiva, el cuerpo humano es considerado como una máquina que puede analizarse desde el punto de vista de sus partes, y la enfermedad no es más que el funcionamiento defectuoso de los mecanismos biológicos que se estudian a través de la biología celular y molecular. Tres siglos después de Descartes, la ciencia médica sigue basándose en el concepto del cuerpo como máquina; de la enfermedad como consecuencia de la avería de la máquina; y de la acción médica como la reparación de esta máquina (5).

La influencia del pensamiento tecno-científico en la medicina contemporánea y los éxitos innegables de la investigación científica, junto a la tecnología contemporánea, han penetrado la cultura de nuestra época y le han otorgado una potencia inigualable al pensamiento cientificista. De esta forma, la ciencia deviene omnipresente y omnipotente, y se presenta como el paradigma del saber que controla, por medio 
de la tecnología, todos los aspectos de la existencia humana (13).

Precisamente por lo anterior, resulta fundamental conocer otras posturas teóricas, que con la fuerza de sus argumentos nos demuestren que no existe una certeza científica absoluta y que todos nuestros conceptos y teorías son limitados y aproximativos. Aceptar la visión de Descartes como verdad absoluta y su método como una manera válida de lograr el conocimiento, ha sido una de las principales causas de nuestro desequilibrio cultural y social. Por lo tanto, sólo será posible encontrar una solución a esta crisis a través de una profunda transformación de nuestras instituciones sociales, de nuestros valores $y$ de nuestras ideas (5).

\section{Otras posturas en torno al concepto de cuerpo- movimiento}

Según Merleau Ponty, el cuerpo y la condición corporal del ser humano implican una decisión acerca de la condición humana. Este gran filósofo planteó que la experiencia de nuestro propio cuerpo invalida el planteamiento dualista moderno al entrañar la unidad originaria de exterioridad e interioridad, extensión y conciencia, materia y sensibilidad. Merleau-Ponty discutió firmemente la opresiva herencia racionalista, desarrollando una fenomenología de la percepción en la cual revaloriza el cuerpo como anclaje de la experiencia. Como afirma este filósofo: "Lo que aparece fenomenológicamente no puede ser ni interior ni exterior, tampoco es apriorísticamente verdadero o falso. Es una fuerte crítica a la tradición dualista occidental, que busca negarle el derecho de ciudadanía al esencialismo de creer en un "verdadero hombre interior" diferenciado de su "fachada falsa" que lo encubre" (14).

El concepto de cuerpo vivido, desde la perspectiva fenomenológico-existencial, deja de lado los reduccionismos orgánicos y/o mentales, rompiendo así con el paradigma mecanicista y abarcando al hombre en su totalidad, sin fragmentarlo en el dualismo cuerpo-mente, sino más bien, definiéndolo como la síntesis del cuerpo y del alma. Aún en los campos de investigación epistemológica de la Fisioterapia, este concepto nuevo permite concebir la corporalidad desde una perspectiva mucho más amplia, una perspectiva desde donde se ubica y comprende la existencia $\mathrm{y}$, por tanto, el movimiento corporal humano (15).

Esta visión reflexiona acerca de la condición desencarnada del sujeto propuesto por la modernidad, en el que, desde el punto de vista objetivo, también el cuerpo es un objeto, y la relación con él y desde él es mediatizada por la reflexión y la experiencia sensible. Ese cuerpo sin carne, el cuerpo como edificio químico o conjunto de tejidos, ese cuerpo "objetivo", es el resultado de un empobrecimiento del fenómeno primordial del cuerpo vivido. De modo que, desde la visión fenomenológica, tiene gran relevancia el análisis de la experiencia íntima del cuerpo propio (16).

"No sólo cuando veo mis manos o cuando me miro en el espejo tengo experiencia de mi propio cuerpo, también cuando desde dentro de él lo siento", explica Pedro Laín Entralgo, quien, en sus escritos sobre antropología médica, expone a su manera los rasgos más importantes de lo que el cuerpo humano es y significa para quien lo vive como suyo. En primera instancia, este autor plantea que el cuerpo, como fundamento de todos los ulteriores decir es, expresa que existimos; y no con referencia al pensamiento cartesiano de "pienso, luego existo" sino como una evidencia anterior a cualquier acto mental. Esta idea significa, entonces, quela experiencia íntima de nuestro propio cuerpo pertenece a la constitutiva necesidad de estar en nuestra existencia, de ser y estar en el mundo; y que todo esto ocurre porque somos corpóreos, porque siendo corpóreos tenemos que estar en el mundo y porque ese mundo tiene presupuesto y fundamento es en nuestra propia corporeidad (17).

En este mismo sentido, el existencialismo de Sartre proporciona una visión ontológica del cuerpo como síntesis indisoluble: mente-cuerpo, cuerpo-conciencia. Visión esta que promueve la humanización del hacer médico con miras a la superación del imperativo biomédico predominante en el ejercicio médico actual. Así, la complejidad y vivencia del cuerpo humano, al igual que su conceptualización, desborda su determinación somática. No se puede ignorar al respecto la capacidad simbólica del cuerpo de relacionar las cosas y los seres, capacidad que ha sido enriquecida con la evolución histórica de la filosofía, las ciencias humanas y sociales y el psicoanálisis (2).

Históricamente, lo sensorial y lo científico han constituido los dos extremos en los que se ha debatido 
el dominio filosófico. La evolución cognitiva e intelectual de la filosofía y la cultura occidental privilegiaron lo segundo en detrimento de lo primero. Por ello se debe empezar por reivindicar el cuerpo no como un opuesto sino como una realidad primordial, lo que implica situar una teoría de los sentidos como el origen de la actividad filosófica (18). Más aún, "cuando no hay modo de comprender el mundo sin detectarlo antes con el radar de los sentidos. Los sentidos no se limitan a darle sentido a la vida mediante actos sutiles o violentos de claridad; desgarran la realidad en tajadas vibrantes y las reacomodan en un nuevo complejo significativo" (19).

Por su parte, Pratima Raichur considera que la vida es la totalidad de la experiencia, y no solamente una colección de componentes físicos. Esta autora plantea que la experiencia humana tiene lugar fundamentalmente a través del filtro de la mente y los sentidos en el nivel de la conciencia, y que la forma como vemos el mundo y lo que sentimos hacia las cosas afecta nuestra experiencia y ésta a su vez cambia el cuerpo (20).

En un sentido similar, Therese Bertherat afirma que: "Nuestro cuerpo es nosotros mismos. Él es nuestra única realidad aprehensible. No se opone a la inteligencia, a los sentimientos, al alma. Los incluye y los alberga. Por ello tomar conciencia del propio cuerpo significa abrirse el acceso a la totalidad del ser...porque cuerpo y espíritu, lo psíquico y lo físico, incluso la fuerza y la debilidad, representan no la dualidad del ser, sino su unidad" (21).

En este sentido, abordamos en particular el paradigma de la complejidad propuesto por Morin. Este autor considera que la prevalencia de un conocimiento fragmentado en las disciplinas, impide realizar el vínculo entre las partes y las totalidades. Insiste en que el ser humano es una unidad compleja de la naturaleza, siendo a la vez físico, biológico, psíquico, cultural, social e histórico. Plantea, en consecuencia, la necesidad de reconocernos a nosotros mismos como seres con una identidad compleja, así como de reconocer a los otros como parte de una identidad compleja común (22).

La complejidad a la que se refiere Morines relación e inclusión, es multidimensional y englobadora. En este sentido, renuncia a un punto de vista único y absoluto para dominar la realidad y el conocimiento. Al contrario, para el pensamiento complejo, es esencial considerar al sujeto y a todos los mecanismos que lo condicionan (23). De ahí que el estudio del cuerpo humano y el movimiento deba realizarse desde un acercamiento holístico y complejo, que permita tomar conciencia de que cada elemento está en relación con otro y que los dos hacen parte de la totalidad, equilibrio necesario para el mantenimiento de la salud del ser.

\section{Enfoque energético del cuerpo-movimiento. Medicina oriental}

Con sus fundamentos filosóficos y teóricos, la medicina oriental brinda un pensamiento del movimiento corporal humano que trasciende el saber científico occidental. La filosofía de Oriente se basa en la premisa de que toda la vida ocurre dentro del ciclo de la naturaleza, de modo que en esta matriz las cosas están conectadas y son mutuamente dependientes. La naturaleza es un sistema unificado en el que sus manifestaciones contrarias se complementan (yinyang). Además, ella está en constante movimiento, siguiendo patrones cíclicos que describen el proceso de la transformación. Cuando los elementos de la naturaleza están en equilibrio, la vida es armoniosa y florece, pero cuando se altera el equilibrio de las fuerzas opuestas, amenaza el desastre.

Desde la visión oriental, el ser humano es un microcosmos de la naturaleza, un universo menor y los seres humanos representan la conexión entre cielo y tierra. En este sentido, la humanidad no puede separarse de la naturaleza: somos naturaleza que se manifiesta como personas. Como un cosmos en miniatura, estamos impulsados por las mismas fuerzas. El bien y el mal son relativos, no absolutos. La vida y la muerte se equilibran entre sí. Lo visible y lo invisible, el soma y la psique son aspectos de un proceso continuo, que siempre cambia y fluye (24). De ahí que la medicina oriental no se halle separada del hombre, sino que surge como necesidad de situarlo en su verdadera dimensión (25).

Más concretamente, para la medicina oriental, el ser humano y por tanto su cuerpo, es un microcosmos, una entidad energética con una determinada forma, estructura y organización. Esta concepción no es difícil de constatar, la ciencia revela como el hombre es un emisor de luz en la gama de los infrarrojos, un cuerpo caliente que depende de la luz del sol para el desarrollo de sus procesos vitales. Se evidencia así que 
el ser humano, como entidad energética que es, adopta una forma y una determinada manera de estructuración y organización,cuya principal característica es el movimiento $(25,26)$.

Como se ve, la medicina oriental hace un paralelismo entre el hombre y la naturaleza. Por consiguiente, los cambios que ocurren en la naturaleza: nacimiento, crecimiento, desarrollo expansión y culminación, operan también en el hombre (25).

En su libro Bioenergética, Alexander Lowen plantea que la cantidad de energía de que dispongamos, y el modo en que la utilicemos, determinan la forma en que respondemos a las situaciones de la vida. Los procesos energéticos están relacionados con el estado de viveza del cuerpo y, por tanto, la rigidez o tensión crónicas pueden disminuir nuestra viveza y menguar nuestra energía (27). El individuo es su cuerpo. No hay persona que exista separada del cuerpo vivo donde está su ser y a través del cual se expresa y se relaciona con el mundo que lo rodea. Para este autor, la mente, el espíritu y el alma son aspectos de todo cuerpo viviente. La persona es la suma total de sus experiencias vitales y las emociones son hechos corporales traducidos en movimientos.

Ahondando en la medicina oriental, se encuentra que la vida depende de tres tesoros: la esencia (jing), la energía (chi) y el espíritu (Shen). La esencia comprende el cuerpo físico de carne y sangre, con todos sus componentes básicos, en particular, los fluidos esenciales: las hormonas, las enzimas y los neurotransmisores. La energía es la fuerza vital original que baña toda célula y todo tejido del cuerpo vivo, activando sus funciones vitales. El espíritu comprende todos los aspectos humanos y primordiales de la mente, entre ellos la conciencia y el conocimiento, el pensamiento y los sentimientos, la voluntad y la intención. Estos tres tesoros funcionan como una sola unidad orgánica (28).

De la medicina oriental hacen parte la acupuntura, el masaje energético, el Tai Chi, las técnicas de acupresión, el yoga, el Ayurveda, entre otras. En nuestra sociedad occidental, todas estas formas médicas son conocidas como terapias alternativas o complementarias; y sobre ellas ha surgido un creciente interés a partir de su creciente uso en diversos países de Europa, África, Asia y América. Los datos de las últimas encuestas realizadas en los Estados Unidos demuestran que más del $40 \%$ de los entrevistados usó alguna terapia alternativa. Además, de acuerdo con la Organización Mundial de la Salud, en los países latinoamericanos también es frecuente el uso de las medicinas complementarias o alternativas. En Chile, por ejemplo, el $71 \%$ de la población ha utilizado alguna vez este tipo de medicinas; lo mismo ha hecho el $40 \%$ de la población colombiana, y entre el $15 \%$ y $20 \%$ de la mexicana. La bibliografía internacional reporta que las personas con trastornos emocionales, principalmente depresión o ansiedad, presentan un uso elevado de las medicinas alternativas y/o complementarias (29).

La medicina oriental propicia una relación más directa y menos fragmentada con los pacientes, ya que toma muy en cuenta la dimensión afectiva y considera al humano como un ser multidimensional; es decir, como una unidad que comprende cuerpo, mente y espíritu. Algo que se ha perdido en los grandes centros hospitalarios, donde el exceso de especialidades hace que el enfermo no llegue a intimar con ningún profesional y se sienta perdido entre tanta ciencia (30), (31).

En virtud de lo expuesto sobre este tema particular, se hace un llamado a los profesionales de la Fisioterapia y de la salud en general para que fijen sus miradas en las medicinas de la tradición oriental. Es evidente el gran aporte que estas ofrecen en la consecución, tan anhelada, del bienestar Psico-físico de nuestra sociedad occidental.

\section{Construcción sociocultural del cuerpo}

$\mathrm{Si}$ durante años la investigación histórica se caracterizó por el silencio sobre el cuerpo, es evidente que en los últimos tiempos su tematización ha generado una importante bibliografía que aborda la cuestión desde diversas perspectivas. Gracias a este proceso, el cuerpo humano ha dejado de ser objeto exclusivo de la biología para ser considerado también una construcción social y cultural.

La histórica vinculación entre las ciencias que estudian el movimiento corporal humano y las ciencias biomédicas ha encumbrado al método científico de las ciencias a un modelo de lo que es "correcto y válido" en nuestro ámbito. La investigación social y sus métodos continúan siendo hoy los grandes desconocidos. Esta soldadura epistemológica de la 
ciencia en torno a lo biomédico se acompaña de una sesgada cultura corporal en nuestra cultura occidental. Existe una tendencia demasiado generalizada a creer que el modelo del cuerpo ofrecido por las referencias biomédicas es unánime y que el resto de significaciones e interpretaciones sobre el mismo carece de criterio alguno. De este modo, algunas posturas teóricas como el yoga, el zen, la acupuntura, el masaje, el chamanismo, se reducen a un puñado de fórmulas ejemplares, de gestos elementales, que flotan en estado de "ingravidez disponibles para cualquier corrupción" (32).

El cuerpo humano es receptor de los acontecimientos sociales y culturales que suceden a su alrededor. También constituye una unidad biológicamente cambiante que por estar en contacto con su entorno se halla sujeto a diversos e importantes significados para la comunicación social (33).

La sociología del cuerpo propone la tesis central de que el cuerpo no es un dato natural o una evidencia inmediata. Trata más bien de mostrar la estructura y la función de los diferentes elementos sociales y culturales que inventan el cuerpo en un tiempo y espacio determinado (34). La cultura nos ofrece un amplio abanico de ideologías sobre el cuerpo y hace de él un espacio privado e íntimo, que sin embargo solo tiene existencia en su intersección con los otros y el mundo. El cuerpo es siempre un signo y, en este sentido, es abordado como modelo de la vida social, donde se valora cada vez más el mundo de sus pasiones y emociones, gestos y hábitos, posturas, instintos y percepciones (35).

La cultura consumista y la comercialización predominantes han convertido al cuerpo humano en uno de sus objetivos, pero además en un vehículo de consumo. De otra parte, el cuerpo se ha considerado como un ítem estadístico yes tratado como una tecnología que hace borroso su límite con las máquinas y los animales (12).

En efecto, durante la modernidad, el cuerpo ha sido presentado a modo de máquina y motor. Analogías que hoy siguen evolucionando e insisten en presentarlo de forma que pueda favorecer el desarrollo masivo de los medios audiovisuales. Esta circunstancia provoca la desaparición del aura que envolvía a los cuerpos humanos. El imaginario del cuerpo ha pasado así por diversas transformaciones y, como ya se dijo, en la actualidad está sometido a la lógica consumista (36).
Las nuevas tecnologías, tan en auge en esta era informática, han transformado completamente nuestras relaciones y nuestra forma de comprender la corporeidad. En efecto, el reciente desarrollo de las innumerables tecnologías biomédicas nos ofrece la posibilidad de transformar el cuerpo hasta lo impensable; nos proporciona la posibilidad de hacer o rehacer nuestros cuerpos en su materialidad según el gusto de cada cual. Esto propicia la creación de nuevos modelos subjetivos en torno a la corporalidad, los cuales mantienen un soporte económico y político en el mercadeo de estas prácticas biotecnológicas, y generan poderosas y nuevas maneras de fabricar sujetos en la cultura occidental. A partir de aquí, el cuerpo sale de sí mismo, adquiere nuevas velocidades y espacios que no son precisamente los que llenan los vacíos producidos por la crisis espiritual del hombre contemporáneo.

Bajo estos patrones de vida, el mundo cotidiano se ve duramente afectado. El acogimiento y el reconocimiento del otro están en crisis. El individualismo reina en la sociedad contemporánea y la soledad es la durarealidad de muchos. En este contexto, cada individuo es un mundo aparte, un mundo que teme encontrarse o juntarse con el otro, y todos los medios establecidos por la tecnología conllevan a esa individualización indiscriminada, como un fenómeno de masas, ambiguo y contradictorio (37).

Esta estructuración social del cuerpo afecta, por una parte, a toda nuestra actividad más inmediata y aparentemente más natural (nuestras posturas, actitudes o movimientos más espontáneos); y, por otra parte, es el resultado tanto de la educación propiamente dicha como de la simple imitación o adaptación. El movimiento inteligente en el ser humano, desde el más simple al más complejo, se aprende culturalmente, incluyendo el sencillo andar cotidiano. Pero la manera de avanzar no es fija ni definitiva en una misma sociedad, sino que puede cambiar y evolucionar según el estilo de vida y los modelos culturales (38).

Le Boulch considera que la manera de descansar, trabajar, caminar o estar de pie difieren de una sociedad a otra, y añade que: "el carácter expresivo del movimiento no es nunca una expresión pura, sino expresión en presencia de los demás, por ende, expresión para los demás. Los movimientos expresivos del cuerpo, sus reacciones tónicas, adquieren una 
dimensión social en la medida en que se revisten de un sentido pragmático o simbólico para los demás" $(38$, 39).

En términos generales, la evolución del tiempo y, con él, de las culturas, permite apreciar una transición del cuerpo prohibido al cuerpo instrumental y, por último, al cuerpo relacional. Las formas de ver el cuerpo dicen mucho de los modelos de comprensión de la realidad que nos orientan, así como de la simbólica general del mundo que nos rodea. No en vano, algunos autores plantean la idea de que es posible comprender la historia de las diversas sociedades humanas investigando tan sólo sus concepciones acerca del cuerpo (39).

En este sentido, el movimiento corporal humano resulta fundamental para la comprensión del objeto de estudio que nos ocupa. Desde este criterio surgen muchos interrogantes: ¿cómo se ha ido transformando el concepto de cuerpo en la Fisioterapia?, ¿podemos pensar en nuevas teorías sociales del cuerpo o la corporeidad?, ¿resulta viable una teoría que fundamente la fisioterapia desde una perspectiva social? Estos interrogantes están a la espera de ser resueltos, de tal forma que avancemos hacia el sentido más humano de la profesión.

También es importante aludir a las distintas maneras de comprensión de la corporeidad que se mueven en los contextos académicos, pues este tema es muy amplio y complejo, y ciertamente no existe una sola mirada con relación a él. En verdad, el concepto de cuerpo va más allá de la misma corporeidad y es fundamental abrirse a la diversidad de enfoques que desde distintas disciplinas enriquecen el hacer profesional del fisioterapeuta.

Con todo, nos hemos olvidado del cuerpo, de su memoria o, lo que es lo mismo, de sus evoluciones y de su presencia en el proceso histórico, circunstancia intolerable cuando observamos que él es la materialidad más contundente $\mathrm{y}$, a la vez, la realidad más inmediata de nuestra entidad psicofísica. El cuerpo se ha adaptado a los usos, costumbres y vaivenes de la historia, y la historia de cada quien pasa por su corporeidad. Los rostros de los seres humanos $\square$ como bien han plasmado artistas y fotógrafos $\square$ dan cuenta y razón de las penalidades, sufrimientos y alegrías que, a través del transcurso del tiempo, dejan su impronta en nuestras facciones (36).
Sin embargo, no debemos caer en el desánimo ni en el pesimismo, ya que existen siempre motivos para la esperanza $y$, justamente, el misterio del cuerpo humano $\square$ más allá de sus analogías con máquinas, motores y robots $\square$ nos invita, una vez más, a trabajar en la configuración de nuevos imaginarios que apuesten a favor de un humanismo integrador, de un humanismo que, sin renunciar al progreso tecnológico, permita un mundo de posibilidades creativas (36).

\section{Perspectivas en torno a la motricidad humana}

El movimiento corporal humano y la motricidad son conceptos que producen muchas controversias por su significado y trascendencia, especialmente en las disciplinas que se orientan a este objeto de estudio. Algunos autores se posicionan sobre la diferenciación clara entre éstos términos. Así, en el concepto de motricidad se clasifican "las características neurocibernéticas que incluyen también factores subjetivos y contenidos de la conciencia". Y ello ocurre así porque la dimensión conceptual de la motricidad excede el simple proceso "espacio-temporal", para situarse en un proceso de complejidad humana: cultural, simbólico, social, volitivo, afectivo, intelectual y, por supuesto, motor. El movimiento es una de las manifestaciones de la motricidad, que lo desborda en complejidad (40).

Hoy existe una nueva perspectiva epistemológica de la motricidad, la cual tiene gran afinidad con los planteamientos que hacen diversos autores desde la complejidad, la fenomenología y la hermenéutica. Ya no resulta suficiente referirse al movimiento como un simple estado de ejecución motriz, sino como un proceso en permanente evolución que contempla al ser desde una perspectiva ontológica; es decir, desde sus propiedades y características más trascendentales( 3 , 41).Entendida así, la motricidad adquiere una dimensionalidad distinta, convirtiéndose en la corporeidad misma (3), en la que, diferentes perspectivas de análisis propuestas desde diferentes objetos formales, intentan obtener conocimiento sobre ella con diferentes fines. La motricidad, como objeto de estudio material, es un sistema compuesto por varios aspectos, a saber: psico-soma-biológicocultural-social. Lo que resulta de esto son manifestaciones práxicas, denominadas manifestaciones expresivas de la motricidad (42). 
Éstas pueden ser abordadas desde diferentes puntos de vista. Jean Le Boulch plantea, por ejemplo, una mutua interdependencia entre la actividad psíquica y la actividad motriz. Su enfoque pretende dar relevancia al desarrollo de las capacidades personales partiendo del movimiento. A partir de aquí, surge el método de la psicokinética que busca principalmente la educación del movimiento y por el movimiento, con el objetivo de lograr un equilibrio físico y mental.

Entonces, Le Boulch configura la psicokinética como una propuesta de educación corporal en la que la educación física desempeña una función transversal en cuanto al papel de la acción motriz para el conocimiento del funcionamiento biológico (38). Desde esta perspectiva, el movimiento corporal humano se aborda en forma más global, considerándolo como una de las dimensiones de la conducta y alejándolo del tradicional dualismo filosófico que nos conduce a la concepción del cuerpo como instrumento. La conducta de un hombre se manifiesta ante el observador a través de sus gestos, movimientos, actitudes corporales, la palabra y la escritura. Por tanto, dicha conducta debe entenderse como "la Ciencia de los actos y movimientos integrados propios de los organismos vivientes evolucionados, en particular, el hombre". El organismo jamás es un sistema en reposo sino siempre el centro de una actividad, la necesidad de acción es la necesidad misma de vivir (39). La experiencia del movimiento se convierte así en la posibilidad del hombre para expresar sus emociones y sentimientos, al tiempo que le permite dar resolución a su propia vida humana (43).

Al respecto aduce John Blofeld I Chinng: "Todo lo que es observable por los sentidos está sometido al cambio y en consecuencia al movimiento... existen ciclos conectados de cambios... no se puede ordenar a los vientos y a las mareas que cesen, pero se puede aprender a navegar en corrientes azarosas si nos conducimos en armonía con los procesos imperantes de la transformación, y así hacer frente a las tormentas de la vida".

En este sentido, apoyamos la construcción de una nueva ciencia de la motricidad que busque respuestas a su verdadero objeto de estudio, sentido y objetivos. Apoyamos una perspectiva de motricidad que se resuelva como proyecto humano, una motricidad caracterizada por la intencionalidad y el significado que le da el sujeto mismo. En otras palabras, un concepto de motricidad en el que converjan distintos puntos de vista con fundamentos claros y que se abran a una influencia recíproca entre ellos.

\section{Teorías en torno al movimiento corporal humano}

Con el fin de aportar al proceso de fortalecimiento y construcción del conocimiento para la Fisioterapia en los ámbitos académico, científico y profesional, han surgido diversas posturas teóricas que fundamentan y sustentan tal objeto de estudio. Con ello se trata de diferenciar el tema entre las distintas ramas del saber y, al mismo tiempo, lograr una comprensión más holística de los conceptos de cuerpo y movimiento. De igual forma, estos diversos abordajes pretenden mostrar el movimiento desde la visión holística, es decir, como un todo que, al mismo tiempo, es el punto de partida por excelencia del comportamiento humano (41).

Los fundamentos teóricos de la Fisioterapia provienen de distintas ramas del saber, principalmente de la filosofía, la historia, la sociología, la física, la anatomía, la fisiología y la cibernética. Estas ramas del saber aportan herramientas conceptuales claves para la observación y el conocimiento del objeto de estudio de la profesión, así como de los factores que contribuyeron a su origen y de aquellos que han determinado su desarrollo (44).

Hoy, la profesión de Fisioterapia, y más específicamente su objeto de estudio, cuenta con un sustento teórico que sirve de marco de referencia para el desarrollo de futuras investigaciones y aportes significativos que den cuenta de la evolución de esta cómo ciencia del movimiento.

En este sentido, la teoría del movimiento continuo propone una teoría general que sirve de base para un cuerpo de conocimientos preciso sobre la profesión y que a su vez es aplicable a la práctica educativa, investigativa y clínica. Esta teoría se propone cumplir con los criterios de globalidad, en el sentido de ser única para la Fisioterapia, de ser lo suficientemente amplia para ser aplicada a todos sus aspectos en la actualidad y en el futuro, así como de servir de base para la práctica educativa e investigativa. Es así como plantea integrar los conocimientos que aportan otras ramas del saber, según perspectivas teóricas específicas. 
El movimiento corporal humano se concibe entonces como un proceso continuo, que incorpora los aspectos físico-patológicos, sociales y psicológicos del ser humano en movimiento. Los principios en los que se basa esta teoría conceptualizan el movimiento como un proceso continuo, que se desarrolla desde un nivel microscópico (molecular) hasta un nivel macroscópico (medio ambiente). Estos niveles de movimiento son influenciados a su vez por factores físicos internos $\mathrm{y}$ externos, como también sociales, psicológicos y ambientales. Precisamente, un aspecto relevante de esta teoría es la consideración de la influencia de factores físicos externos y sociales para el desarrollo del movimiento corporal humano (45).

De igual forma, el ambiente social tiene múltiples influencias sobre el movimiento individual, debido principalmente a la diversidad de normas y de roles culturales, que influyen de manera definitiva en la motivación, frecuencia y calidad del movimiento. Con otras palabras, esta teoría plantea que los aspectos sociales, como la política, economía y legislación en cuanto a accesibilidad y elegibilidad de programas de salud y otros servicios sociales, tiene un impacto trascendental sobre el movimiento corporal humano.

La anterior teoría se relaciona con el modelo ecológico propuesto por Urie Bronfenbrenner, quien destaca la importancia de estudiar los ambientes en los que actuamos. El autor de este modelo ofrece una perspectiva teórica para la investigación del desarrollo humano que plantea una nueva concepción de la persona en desarrollo y del ambiente que la rodea, pero especialmente de la interacción entre ambos. Es por esto que Bronfenbrenner define el desarrollo como un cambio perdurable en el modo en que una persona percibe su ambiente y se relaciona con él (46).

$\mathrm{El}$ ambiente ecológico se concibe como un conjunto de estructuras seriadas, cada una de las cuales cabe dentro de la siguiente. En el nivel más interno se encuentra el entorno inmediato que contiene a la persona en desarrollo. El siguiente nivel conduce fuera del espacio conocido, pues más allá de cada entorno por separado, nos obliga a analizar las relaciones que existen entre ellos, y estas interconexiones pueden ser tan decisivas para el desarrollo como lo que sucede dentro de un entorno determinado. El tercer nivel del ambiente ecológico lleva aún más lejos: plantea la hipótesis de que el desarrollo de la persona se ve afectado profundamente por hechos que ocurren en entornos en los que ella ni siquiera está presente (46).
Así, según la teoría del movimiento continuo, cada nivel de movimiento está influenciado por los niveles de movimiento que lo preceden y suceden, de modo que las interacciones que se producen entre éstos son dinámicas. Para las autoras de la teoría del movimiento continuo, lo esencial en la Fisioterapia consiste en entender que el movimiento tiene propiedades $\mathrm{y}$ relaciones que van desde un nivel micro hasta uno macro, y que éstos dependen de la habilidad del individuo para moverse en cada nivel del movimiento continuo (Movimiento Potencial Máximo AdquiribleMPMA). A su vez, este movimiento puede estar determinado por las condiciones biológicas, psicológicas y sociales de cada persona, así como de las relaciones entre estos factores.

Otros conceptos importantes para la teoría del movimiento continuo son la Capacidad de Movimiento Preferida (CMP) y la Capacidad de Movimiento Corriente (CMC). Éstas hacen referencia a los niveles en que algunos individuos funcionan de forma confortable para satisfacer sus necesidades básicas diarias; es así como, dentro de los límites establecidos por el MPMA, las características particulares de cada individuo junto con el ambiente y la sociedad en que vive, tendrá una capacidad preferida y corriente de movimiento para cada nivel del continuo. Desde este punto de vista, el objetivo de la Fisioterapia es minimizar la diferencia entre la CMP y la CMC y así aumentar el movimiento. El objetivo de Esta teoría es proporcionar una base teórica para que el modelo de tratamiento sea desarrollado en varias áreas de la práctica (45).

Por último, encontramos la teoría del movimiento como sistema complejo. Esta teoría plantea una referencia para el movimiento corporal humano desde el concepto de sistema complejo, el cual pone de relieve la autoestructuración de un sistema con relación a niveles, subsistemas y componentes que establecen múltiples relaciones con grados de complejidad diferentes (47). Así se hace manifiesta la necesidad de recuperar la unidad preexistente a todo análisis científico. Necesidad ésta que no desprecia la subjetividad sino que, por el contrario, se afirma en la concepción unitaria de cuerpo y movimiento, así como de las polaridades tensión-relajación, parte-todo, movimiento-estatismo, emoción-acción, fuerzasuavidad, entre muchas otras, las cuales expresan el reconocimiento de los opuestos complementarios propuestos por Morin, que las tradiciones de Oriente 
venían planteando en su teoría del yin y el yang u opuestos complementarios desde la alta antigüedad.

Con base en estas características, el movimiento humano dejaría de existir como la sumatoria de las áreas físicas, motoras, orgánicas, funcionales $\mathrm{y}$ cognitivas,y más bien se centraría en la lectura de un entretejido entre lo objetivo y lo subjetivo (9). Para los autores de ésta teoría, el movimiento como sistema complejo presenta tres niveles de interacción en los procesos, que determinan el grado de relación, interacción y delimitación con el entorno. Entre estos niveles, el control motor constituye el primer nivel de interacción y está relacionado con la capacidad motora del individuo, que a su vez integra sus componentes biológicos, psicológicos y sociales. De esta manera, el control motor permitiría reorganizar la actividad motora y determinaría sus características. El segundo nivel de interacción es el aprendizaje motor. Éste integra las transformaciones permanentes de los patrones de movimiento desde la perspectiva particular e individual, poniendo en juego la capacidad motora (potencialidades del hombre) y el control motor. Por último, el tercer nivel de interacción es constituido por el contexto, considerado como el escenario temporoespacial que condicionan la capacidad motora del individuo a partir de normas y reglas sociales (47). Esta teoría se encuentra acorde con el paradigma de la complejidad, en el cual no es posible explicar o comprender una realidad particular sin comprender las múltiples relaciones que determinan la totalidad.

\section{Conclusiones}

Con base en lo anteriormente expuesto, podríamos resaltar la siguiente conclusión:

En la medicina actual, resultan clarísimas las consecuencias negativas de la visión reduccionista. El pensamiento médico, suscrito a la imagen cartesiana del cuerpo humano como un mecanismo de relojería, no puede entender muchas de las principales enfermedades crónicas presentes en el mundo de hoy; y aunque el propio Descartes reconoció que no podía llevar a término su filosofía científica, el método de razonamiento y el esquema general de la teoría sobre los fenómenos naturales han determinado el pensamiento científico de Occidente durante los últimos cuatro siglos. Las prácticas médicas basadas en este enfoque parcial no resultan muy eficaces en la promoción y el mantenimiento de la salud integral del individuo, y esta situación no cambiará mientras la ciencia médica no relacione el estudio de los aspectos biológicos de la enfermedad con la condición psicológica del organismo humano y con su entorno (5).

Asimismo, resultan evidentes los pocos esfuerzos de la mayoría de las disciplinas relacionadas con este objeto de estudio $\square$ el movimiento corporal humano $\square$ y de algunos investigadores por reconocer el "carácter emocional del movimiento" (2). Dichas disciplinas deben profundizar en una comprensión del ser humano desde su acción-comportamiento, es decir, realizar un análisis sobre la configuración y construcción de los procesos que permitan expresar su condición y su posibilidad de acción, de conformidad con los sentires de la existencia humana, pero al mismo tiempo en un despliegue relacional (12).

El paradigma mecanicista del cuerpo está en crisis y necesita ser replanteado. Este paradigma ha marcado el desarrollo de la Fisioterapia hasta nuestros días, provocando un estancamiento del desarrollo de la misma, ya que sólo ve el cuerpo como un objeto distante y susceptible de ser manipulado. En medio de esta crisis, comienzan a surgir nuevas lecturas sobre la corporeidad, que la han convertido en escenario de diferentes vertientes disciplinarias y transdisciplinarias.

Por otro lado, un nuevo enfoque sobre los conceptos cuerpo-movimiento propiciará el rescate de la verdadera identidad del ser-individuo $\square$ en relación con el otro $\square$, pues, en última instancia, el individuo depende siempre del sentido de los otros, ya que son los otros quienes nos reconocen y distinguen.

La identidad se construye en la interacción social, y el cuerpo participa de esta relación social, por lo que es uno de los núcleos centrales en la formación de la identidad (48). Podría establecerse, entonces, una nueva postura paradigmática, una postura que recupere el sentido del movimiento corporal humano desde una mirada antropológica, energética, sociológica y ontológica. Todo esto con el objetivo de encarar el sufrimiento humano de la manera más respetuosa, y esta cuestión exige que la integridad e individualidad del ser sea un asunto prioritario (13).

Necesitamos una nueva medicina, en la que la fuerza que ocasiona la enfermedad sea la misma que la cura: en la que una persona y su enfermedad se unan mediante la responsabilidad: una medicina en la que 
cuerpo y mente sean una entidad única e indistinguible (49). Esta nueva medicina no considerará a las emociones como hechos fugaces ni aislados en el espacio mental, sino como expresiones de la conciencia, materia fundamental de la vida expresada a través del cuerpo (50).

De igual manera, la existencia de propuestas innovadoras como las planteadas por las teorías del movimiento continuo y el movimiento como sistema complejo resultan alentadoras. Su relación con el modelo ecológico y el enfoque holístico planteado por la medicina oriental abren nuevas vías para la Fisioterapia del siglo XXI. Cabría entonces esperar su influencia en los programas de Fisioterapia, para ponernos a tono con los nuevos vientos que impulsan el estudio del movimiento corporal humano

\section{Referencias}

1. Ce L, Ve J, Cordero NL, Olivieri FJ. Los filósofos presocráticos. Heráclito, Parménides, Zenón de Elea, Meliso de Samos. Madrid: Planeta; 1998.

2. Triana JE. Dimensiones ontológicas del cuerpo. Bogotá: Ediciones el Bosque; 1997.

3. Prieto RA, Naranjo PS, García SL. Cuerpo Movimiento: Perspectivas. Colección de textos rehabilitación y desarrollo humano. Bogotá: Universidad del Rosario; 2005.

4. Raichur P, Cohn M. Belleza Verdadera, Bogotá: Norma; 1998.

5. Capra F. El punto crucial. Ciencia, sociedad y cultura naciente. Argentina: Estaciones; 1998.

6. Cely CS, Mogollón MA. Caracterización de algunas características del movimiento corporal humano. Revista ASCOFI. 2000; (45): 62-65.

7. Solana RJ, Morin E. Por un pensamiento complejo. Implicaciones interdisciplinares. Madrid: Akal; 2005.

8. Toro JA. El cuerpo en evidencia: Reflexiones sobre aspectos sociales y clínicos de la corporalidad. Rev. puertorriq. psicol. 2007; (18): 77-81.

9. Pérez MH. Cuerpo Humano, Campo de conocimiento. Relaciones. 2009; 30 (117): 11-19.
10. Descartes R. El discurso del método. Alicante (España): Club Universitario; 2005.

11. Arango PC. Movimiento corporal humano y salud. Educación y Universidad. 2002; 2(6): 7678.

12. Escobar TJ. La globalización del paradigma biomédico: biotecnología y ciencia vs. la medicina como profesión histórica. Revista Colombiana de Bioética. 2010; 5(2): 94-102.

13. Stagnaro JC. Bioética, formación de los médicos y ejercicio de la medicina en el marco del reduccionismo biomédico y la globalización económica. Investigación en salud. 2002; 4(002): 0-8.

14. Bech JM, Ponty M. Una aproximación a su pensamiento. Barcelona: Anthropos; 2005.

15. De Castro A, García G. La dimensión corporal desde el enfoque fenomenológico - existencial. Psicología desde el Caribe. 2006; 017:122-148.

16. Pfeiffer ML. Cuerpo y finitud. Una cuestión definitiva en las filosofías de Merlau Ponty y Nietzhze. Enfoques. 2008; 20 (1-2): 47-72.

17. Laín EP. Antropología Médica. Madrid: Salvat; 1984.

18. Ayús RR, Eroza SE. El cuerpo y las ciencias sociales. Revista pueblos y frontera digital. 2008; 004: 1-56.

19. Ackerman D. Una historia natural de los sentidos. Barcelona: Anagrama; 2000.

20. Raichur P, Cohn M. Belleza verdadera. Bogotá: Norma; 1998.

21. Therese B, Cernstein C. El cuerpo tiene sus razones. Barcelona: Paidós Ibérica; 2006.

22. Morín E. Los siete saberes necesarios para la educación del futuro. Barcelona: Paidós Ibérica; 2001.

23. Morín E, Soto González M; Complejidad y sujeto humano. Valladolid (España): Universidad de Valladolid; 1999.

24. Beinfield H, Korngold E. Entre cielo y tierra. Los cinco elementos en la medicina china. Barcelona: Los libros de la liebre de marzo; 1999. 
25. Padilla Corral JL. Tratado de sanación en el arte del soplo. Madrid: Escuela Neijing; 1999.

26. Lowen A. Bioenergética. México: Diana: 1979.

27. Lowen A, Lowen L. Ejercicios de Bioenergética. Málaga (España): Sirio; 2003.

28. Reid D. Los tres tesoros de la salud. Barcelona: Urano; 1993.

29. Gorn BS, Navarro A, Saavedra S, Solano N. El uso de las terapias alternativas y complementarias en población mexicana con trastornos depresivos y de ansiedad: Resultados de una encuesta en la ciudad de México. Salud mental. 2009; 32 (2); 107-115.

30. Arrea BC. Paradojas de la medicina actual. Acta médica costarricense. 2009; 51 (39); 136-137.

31. Hernández AA, González RL, Rocha CD. Manejo del dolor en una Cervicalgia a través de la acupuntura como un coadyuvante en la intervención fisioterapéutica. Umbral científico. 2008; 012: 81-89.

32. Le Breton D. Antropología del cuerpo y modernidad. Buenos Aires: Nueva Visión; 2002.

33. Acuña DA. El cuerpo en la interpretación de las culturas. Boletín Antropológico. 2001 1(51): 31 52.

34. Zapata CR. La dimensión social y cultural del cuerpo. Boletín de antropología. 2006; 20(037): 251-264.

35. Escarano L. Palabras en el cuerpo. Literatura y experiencia. Buenos Aires: Biblos; 2007.

36. Conrad V. La configuración postmoderna del cuerpo humano. Movimiento. 2000; 7(13): 81-98.

37. Camps V. Una vida de calidad: Reflexiones sobre bioética. Barcelona: Crítica; 2001.

38. Chaverra BE, Fernández I, Uribe PD. Aproximaciones epistemológicas y pedagógicas a la Educación Física. Un campo en construcción. Medellín; 2007.

39. Acuña DA. El cuerpo en la interpretación de las culturas. Boletín Antropológico. 2001; 1(51): 31 52.

40. De Morais ML, Gomes LN, Dantas MG, Villaverde GC. Estudio antropo-epistemológico de la motricidad humana y reflexiones para las ciencias del movimiento. Scientia. 2009; 14(1): 43-63.

41. Rebel G. El lenguaje corporal. Lo que expresan las actitudes, las posturas, los gestos y su interpretación. Madrid: Edaf; 2004.

42. Rey A, Canales I. Discurso epistémico para una ciencia de la motricidad. Cinta de Moebio. 2007; 028: 104-123.

43. Schneider ER. El movimiento en la vida cotidiana: el valor de la experiencia corporal. Revista Digital - Buenos Aires. 2004; 10 (72):115.

44. Chillón MJ, Rebollo RA, Meroño GJ. El pensamiento histórico-filosófico y los fundamentos científicos en el estudio de la fisioterapia. Rev. Fisioter. 2008; 7 (2): 5-16.

45. Cott CA, Finch E, Gasner D, et al. The movement continuum theory of physical therapy. Physiother Can.1995; 47:87-95.

46. Bronfenbrenner U. La ecología del desarrollo humano. Barcelona: Paidós transciciones; 2002.

47. Triana JA, Arenas QB, Restrepo BH, Toro DJ, Rodríguez GJ, Hoover VJ, et al. El movimiento como sistema complejo. Revista digital Buenos Aires. 2000; 5(26).

48. Sánchez MJ. La comunicación sin cuerpo. Identidad y virtualidad. Revista mexicana de ciencias políticas y sociales. 2010; 52(209): 37 51 .

49. Hammer L. Psicología y Medicina China. Barcelona: Ediciones la Liebre; 2002.

50. Chopra D. Cuerpos sin edad, mentes sin tiempo. Barcelona: Javier Vergara Editor; 1998. 